\title{
¿Qué patrón de Doppler se puede encontrar en la miocardiopatía hipertrófica?
}

Roberto Flórez Gómez*

Laura López Maldonado**

* Técnico Imagen Cardíaca. Hospital Universitario La Paz. Madrid. España

** Técnico Imagen Cardíaca. Hospital Universitario 12 de Octubre. Madrid. España

\section{Correspondencia}

Roberto Flórez Gómez

florezgomezroberto@gmail.com

Recibido: 30/05/2020

Aceptado: 30/05/2020

En línea: 31/07/2020

Citar como: Flórez-Gómez R, López-Maldonado L. ¿Qué patrón de Doppler se puede encontrar en la miocardiopatía hipertrófica? Rev Ecocar Pract (RETIC). 2020 (Jul); 3 (2): 48-50. doi: 10.37615/retic.v3n2a15

Cite this as: Flórez-Gómez R, López-Maldonado L. Doppler pattern in hypertrophic cardiomyopathy. What we must look at? Rev Ecocar Pract (RETIC). 2020 (Jul); 3 (2): 48-50. doi: 10.37615/retic.v3n2a15.

\section{Palabras clave \\ $\triangleright$ Miocardiopatía hipertrófica \\ $\triangleright$ Doppler \\ $\triangleright$ Ecocardiografía}

\begin{tabular}{l} 
Keywords \\
\hline$\triangleright$ Hypertrophic \\
$\quad$ cardiomyopathy \\
$\triangleright$ Doppler \\
$\triangleright$ Echocardiography \\
\hline
\end{tabular}

\section{RESUMEN}

Se describirá brevemente el método más aceptado para el diagnóstico ecocardiográfico en pacientes con miocardiopatía hipertrófica. Es importante distinguir entre los diferentes patrones de Doppler que se pueden encontrar en la práctica ecocardiográfica diaria.

ABSTRACT
In the following article we will briefly describe the most accepted method for echocardiographic diagnosis in pa-
tients with hypertrophic cardiomyopathy. It will be important to distinguish between the different Doppler pat-
terns that we can find in daily echocardiographic practice.

La miocardiopatía hipertrófica (MCH) se define por la presencia de un aumento del grosor de la pared del ventrículo izquierdo (VI) que no se puede explicar únicamente por condiciones de carga anómalas.

En un paciente adulto, la MCH se define por un grosor de la pared mayor o igual a $15 \mathrm{~mm}$ en uno o más segmentos miocárdicos del VI determinados por cualquier técnica de imagen ${ }^{(1)}$

La ecocardiografía es una de las técnicas de referencia en el diagnóstico, seguimiento y pronóstico de esta enfermedad. Los puntos clave en este sentido son los siguientes ${ }^{(2)}$ :

- Grosor de la pared del VI. Se debe medir en telediástole. Es esencial que se evalúen todos los segmentos desde la base hasta el ápex ${ }^{(3)}$, asegurándose de que se registran los grosores a nivel de la válvula mitral (segmentos 1 a 6), nivel medioventricular (segmentos 7 a 12) y apical en los planos de eje corto (segmentos 13 a 16)

En los planos apicales se puede evaluar el grosor del segmento 17 (ápex).

- Tamaño de la aurícula izquierda. Su tamaño aporta importante información pronóstica. Suele encontrarse dilatada debido a la insuficiencia mitral relacionada con el movimiento sistólico anterior y las presiones de llenado elevadas.

- Función diastólica. Incluyendo imagen miocárdica (Doppler tisular), velocidades del flujo de las venas pulmonares y presión sistólica de la arteria pulmonar.

Valoración del ápex, y cribado de trombos apicales.
Aproximadamente un tercio de los pacientes tiene en reposo movimiento sistólico anterior de las valvas de la válvula mitral que produce obstrucción del tracto de salida del VI, mientras que otro tercio tiene la obstrucción sólo durante las maniobras que cambian las condiciones de carga y contractilidad del ventrículo (Valsalva).

Este movimiento puede producirse por varios factores, entre los cuales se encuentran el engrosamiento-hipertrofia del septo basal, la inserción o disposición anormal de los músculos papilares y anomalías intrínsecas de las valvas mitrales (Vídeo 1).

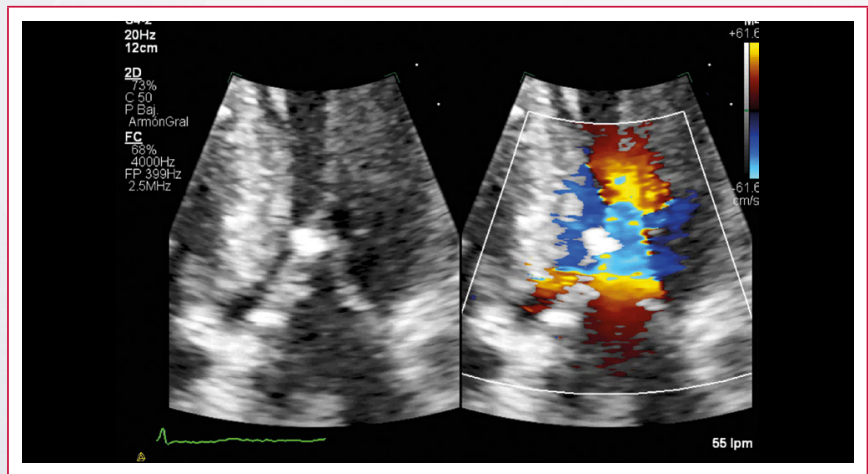

Vídeo 1. Imagen comparativa de un plano de cinco cámaras con color para visualizar el artefacto de aliasing por el aumento de velocidad en el tracto de salida del ventrículo izquierdo 


\section{- Trukipedia \\ truco 02}

Para valorar mejor el tracto de salida del VI, se debe visualizar anatómicamente la imagen bidimensional. Se puede utilizar la caja de color ajustada al VI, y rastrear (desde el ápex hasta la base) con el Doppler pulsado para situar el punto exacto donde dicha obstrucción se está produciendo (Figura 1). Se utilizará el Doppler continuo para medir el gradiente de obstrucción.

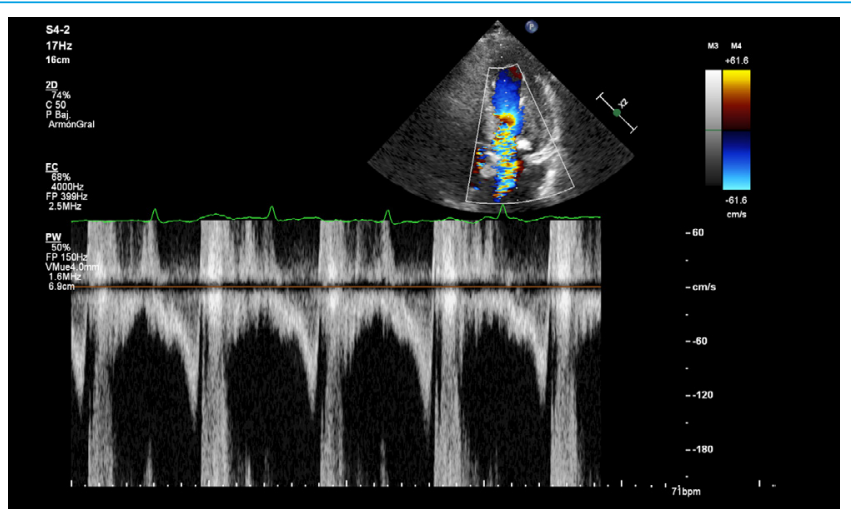

Figura 1. Imagen de Doppler pulsado, a nivel del VI, para identificar a qué nivel se encuentra la obstrucción. Flujo con forma de daga. También se puede utilizar el Doppler color

El patrón de Doppler continuo a través del tracto de salida del VI tiene forma de daga, esto es porque a medida que avanza la sístole va aumentando la presión (gradiente dinámico) (Figura 2). Esta forma hace que se diferencie fácilmente del patrón de estenosis aórtica, que como la estrechez es constante a lo largo de la sístole el patrón de Doppler es simétrico (Figura 3).

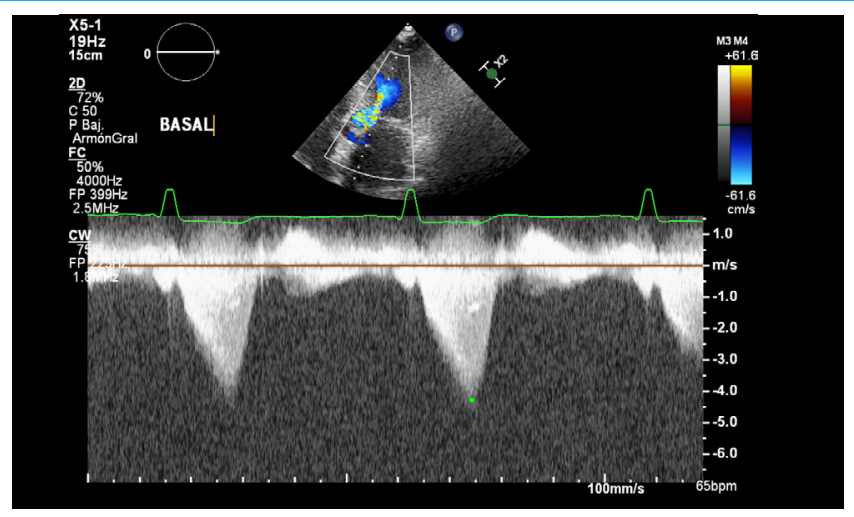

Figura 2. Imagen de Doppler continuo a través del tracto de salida del VI, con forma de daga y un gradiente máximo de $73 \mathrm{mmHg}$, alcanzando el pico máximo al final de la sístole

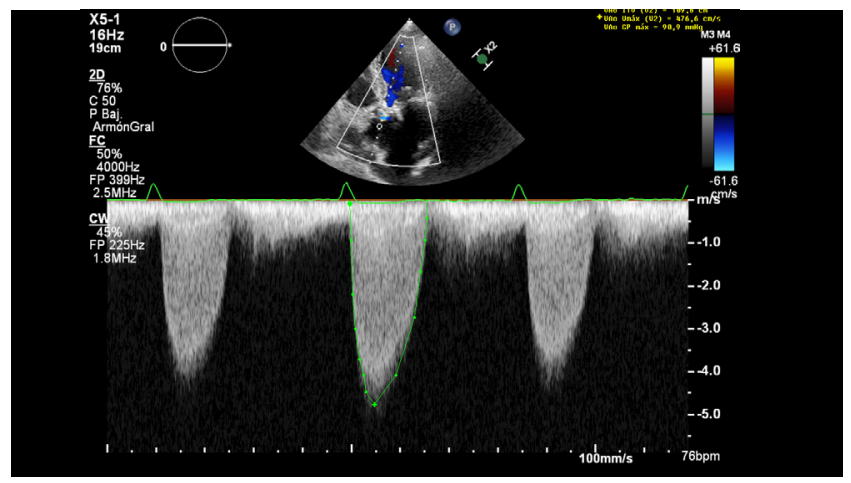

Figura 3. Imagen de Doppler continuo sobre la válvula aórtica, donde se visualiza el patrón de estenosis aórtica, pudiendo calcular un gradiente máximo de $90 \mathrm{mmHg}$ y un gradiente medio de $54 \mathrm{mmHg}$
Por convención, se define gradiente de obstrucción dinámico al mayor de $30 \mathrm{mmHg}$ en reposo o durante la provocación fisiológica en la maniobra de Valsalva, al ponerse de pie o con el ejercicio. Cuando el gradiente supera los $50 \mathrm{mmHg}$ se considera hemodinámicamente significativo (Figura 4).

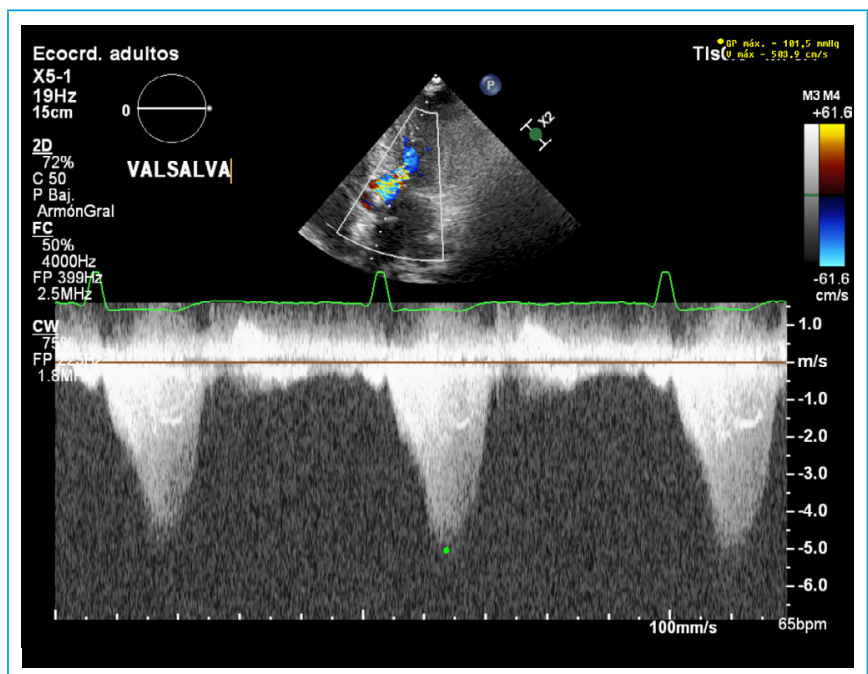

Figura 4. Imagen de Doppler continuo a través del tracto de salida del V del mismo paciente de la Figura 2, tras solicitarle que realice la maniobra de Valsalva. El gradiente aumenta hasta $101 \mathrm{mmHg}$

Es importante diferenciar la presencia de obstrucción no relacionada con el movimiento sistólico anterior, incluyendo las membranas subaórticas, las anomalías de las valvas de la válvula mitral y la obstrucción medioventricular, sobre todo cuando se prevé una cirugía.

Normalmente, cuando existe el movimiento sistólico anterior de la válvula mitral, se suele producir un fallo de coaptación de las valvas e insuficiencia mitral, que es típicamente de meso-telesistólica (Vídeo 2).

Es importante diferenciar la velocidad y el tiempo de chorro mitral, de la turbulencia por la obstrucción del tracto de salida del ventrículo izquierdo (Figura 5).

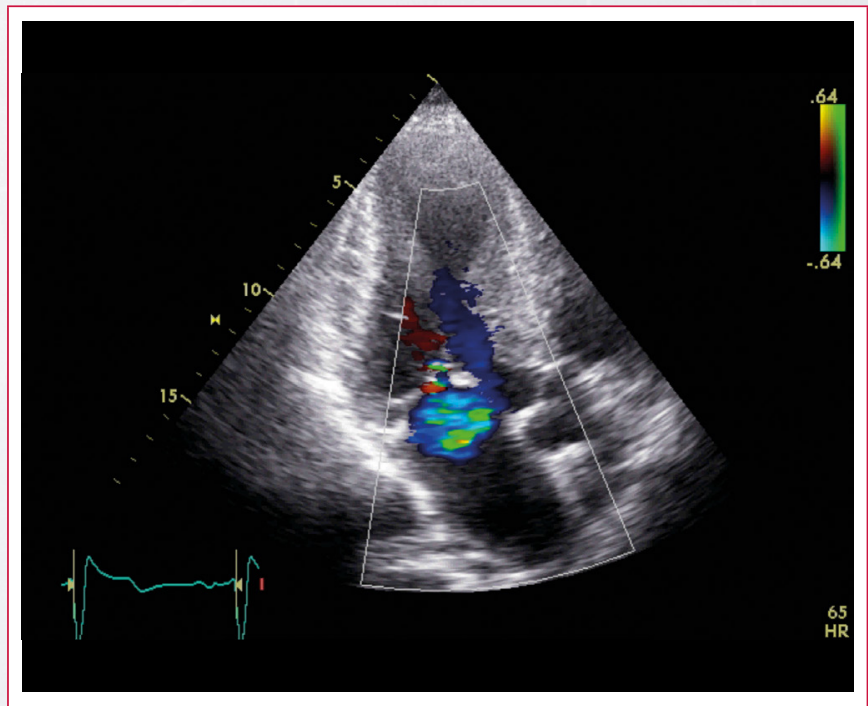

Vídeo 2. Imagen del plano de tres cámaras con color, donde se visualiza el aliasing por aumento de velocidad a nivel del tracto de salida del VI, y la insuficiencia mitral excéntrica con jet posterolateral debido al movimiento sistólico anterior de la válvula mitral (SAM) 


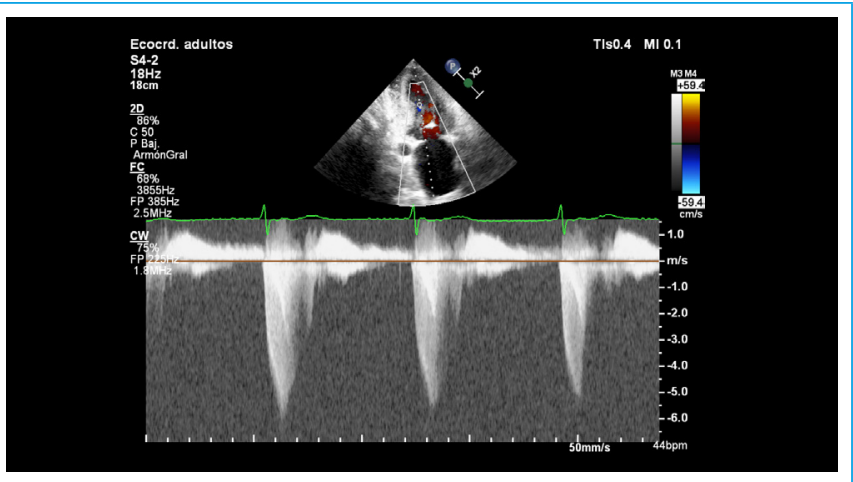

Figura 5. Imagen de Doppler continuo sobre el plano dos cámaras donde se representan en el mismo espectro de Doppler las dos señales de flujo. Gradiente con forma de daga (con velocidad más baja en forma de daga) y gradiente de flujo de insuficiencia mitral (protosistólico)

La insuficiencia mitral es de naturaleza dinámica y su intensidad y duración a lo largo de la sístole varia con el grado de severidad (Figura 6).

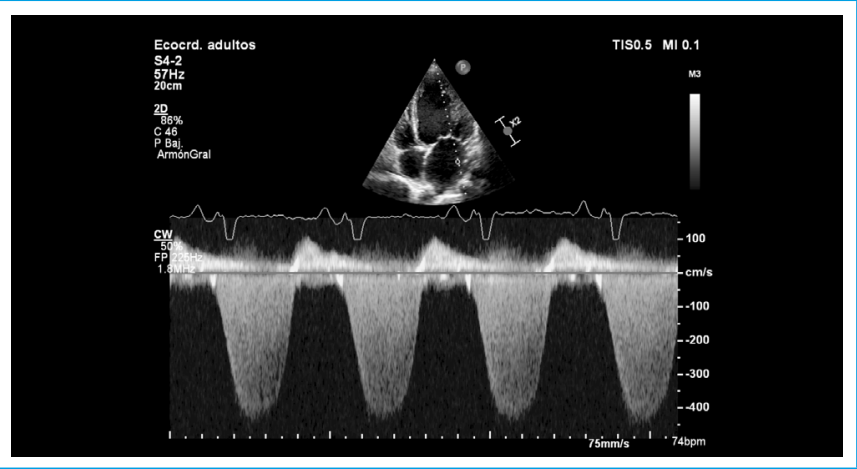

Figura 6. Imagen de Doppler continuo sobre válvula mitral con el patrón del flujo de insuficiencia mitral severa holosistólica

\section{Ideas para recordar}

- Es importante evaluar todos los segmentos del ventrículo izquierdo para evaluar el engrosamiento del miocardio utilizando el eje corto y los planos apicales.

- Rastrear el tracto de salida del ventrículo izquierdo para localizar mejor el máximo gradiente basal y con maniobra de Valsalva utilizando el Doppler continuo, ajustando la escala de velocidad.

- El gradiente de obstrucción será mayor al final de la sístole, debido al aumento de presión, y por eso adquiere una forma de daga.

\section{Bibliografía}

1. Elliott PM, Anastasakis A, Borger MA, et al. Guía de practica clínica de la ESC 2014 sobre el diagnóstico y manejo de la miocardiopatía hipertrófica. Rev Esp Cardiol 2015; 68: 63.e1-e52.

2. Martín J, Solís J, Cruz I, et al. Manual de Imagen en Cardiología. ISBN: 13: 97884-86671-83-9.

3. Cerqueira MD, Weissman NJ, Dilsizian V, et al. Standardized myocardial segmentation and nomenclature for tomographic imaging of the heart. Circulation 2002; 105: 539-542. 